\title{
IL-22 and its receptors are increased in human and experimental COPD and contribute to pathogenesis
}

\author{
Malcolm R. Starkey ${ }^{1}$, Maximilian W. Plank¹, Paolo Casolari², Alberto Papi (1) ${ }^{2}$, \\ Stelios Pavlidis ${ }^{3}$, Yike Guo ${ }^{3}$, Guy J.M. Cameron ${ }^{1}$, Tatt Jhong Haw ${ }^{1}$, \\ Anthony Tam ${ }^{4,5}$, Ma'en Obiedat ${ }^{4,5}$, Chantal Donovan ${ }^{1}$, Nicole G. Hansbro ${ }^{1,6,7}$, \\ Duc H. Nguyen ${ }^{1}$, Prema Mono Nair ${ }^{1}$, Richard Y. Kim ${ }^{1}$, Jay C. Horvat ${ }^{1}$, \\ Gerard E. Kaiko ${ }^{1}$, Scott K. Durum ${ }^{8}$, Peter A. Wark ${ }^{1}$, Don D. Sin ${ }^{4,5}$, \\ Gaetano Caramori (10 ${ }^{9}$, Ian M. Adcock ${ }^{3}$, Paul S. Foster ${ }^{1}$ and Philip M. Hansbro ${ }^{1,6,7}$
}

@ERSpublications

IL-22 and its receptors are increased in both human and experimental chronic obstructive pulmonary disease (COPD). IL-22 drives neutrophilic inflammation and impaired lung function in experimental COPD. http://bit.ly/2Vsri6T

Cite this article as: Starkey MR, Plank MW, Casolari P, et al. IL-22 and its receptors are increased in human and experimental COPD and contribute to pathogenesis. Eur Respir J 2019; 54: 1800174 [https:// doi.org/10.1183/13993003.00174-2018].

ABSTRACT Chronic obstructive pulmonary disease (COPD) is the third leading cause of morbidity and death globally. The lack of effective treatments results from an incomplete understanding of the underlying mechanisms driving COPD pathogenesis.

Interleukin (IL)-22 has been implicated in airway inflammation and is increased in COPD patients. However, its roles in the pathogenesis of COPD is poorly understood. Here, we investigated the role of IL22 in human COPD and in cigarette smoke (CS)-induced experimental COPD.

IL-22 and IL-22 receptor mRNA expression and protein levels were increased in COPD patients compared to healthy smoking or non-smoking controls. IL-22 and IL-22 receptor levels were increased in the lungs of mice with experimental COPD compared to controls and the cellular source of IL-22 included $\mathrm{CD} 4^{+} \mathrm{T}$-helper cells, $\gamma \delta \mathrm{T}$-cells, natural killer T-cells and group 3 innate lymphoid cells. CS-induced pulmonary neutrophils were reduced in IL-22-deficient $\left(I l 22^{-l-}\right)$ mice. CS-induced airway remodelling and emphysema-like alveolar enlargement did not occur in $I l 22^{-1-}$ mice. $I l 22^{-1-}$ mice had improved lung function in terms of airway resistance, total lung capacity, inspiratory capacity, forced vital capacity and compliance.

These data highlight important roles for IL-22 and its receptors in human COPD and CS-induced experimental COPD. 


\section{Introduction}

Chronic obstructive pulmonary disease (COPD) is the third leading cause of morbidity and death and imposes a significant socioeconomic burden globally [1]. It is a complex, heterogeneous disease characterised by chronic pulmonary inflammation, airway remodelling and emphysema, which are associated with progressive lung function decline [2]. Cigarette smoke (CS) is a major risk factor for COPD [2]. The mainstays of therapy for COPD are glucocorticoids, $\beta_{2}$-adrenergic receptor agonists and long-acting muscarinic antagonists [3]. However, these agents only provide symptomatic relief rather than modifying the causal factors or suppressing disease progression [3]. There is emerging interest in altered lung and gut microbiomes and the gut-lung axis which could be modified for therapeutic gain $[4,5]$. However, there is currently a lack of effective treatments for COPD due to the poor understanding of the underlying mechanisms.

Interleukin (IL)-22 is a member of the IL-10 cytokine family which is implicated in several human diseases, including mucosal-associated infections and inflammatory disorders of the lung [6]. CD4 ${ }^{+}$ T-helper cells, $\gamma \delta$ T-cells, natural killer (NK)T-cells and group 3 innate lymphoid cells (ILC3) are generally the major cellular sources of IL-22 [6]. Unlike IL-22, expression of the IL-22 receptor (IL-22R) is largely restricted to structural cells. This ligand-receptor distribution permits immune cells to regulate responses of stromal cells, and particularly at barrier surfaces such as the lung, where epithelial cells play an active role in initiating, regulating and resolving immune responses. IL-22R is a cell-surface heterodimer consisting of IL-22RA1 and IL-10RB [6]. IL-22RA2 is a naturally occurring IL-22 antagonist which negatively regulates IL-22-induced inflammatory responses [6,7]. Functional studies in murine systems indicate that IL-22 has immune-regulatory properties in infection, inflammation, autoimmunity and cancer [6]. In these models, the functional consequences of IL-22 expression can be either pathological or protective, depending on the context in which it is expressed. Indeed, increased IL-22 levels and IL-22 $2^{+}$ cells have been demonstrated in the blood, sputum and lung biopsies of COPD patients [8]. The role of IL-22 in lung antimicrobial defence and the impact of COPD on this defence pathway has been reported $[9,10]$. In experimental COPD, Haemophilus influenzae infection impaired IL-22 production, and wild-type (WT) and IL-22-deficient $\left(^{-1-}\right)$ mice had impaired clearance [10]. CS exposure suppressed Streptococcus pneumoniae-induced IL-22 production and treatment with recombinant IL-22 restored bacterial clearance [11]. Despite this, there is limited knowledge of the role that IL-22 plays in COPD pathogenesis independent of respiratory infection.

Here, we investigate its role using gene expression analysis of airway epithelial brushings and parenchymal cores from human COPD patients, an established mouse model of CS-induced experimental COPD that recapitulates the critical features of human disease [4, 12-18], and IL-22 reporter and Il22-/- mice [19]. IL-22 and IL-22R mRNA and protein were increased in the airways of mild-to-moderate COPD patients. IL-22 and IL-22 ${ }^{+}$T-cells and ILC3s were increased in experimental COPD. CS-induced pulmonary neutrophilic inflammation, airway remodelling and emphysema were reduced and lung function was improved in $I l 22^{-/-}$mice compared to WT controls, thus implicating IL-22 in COPD pathogenesis.

\section{Methods}

Ethics statement, animal details, additional methods and statistical analyses are described in the supplementary material.

\section{Human gene expression}

Analysis of IL22, IL22RA1, IL10RB and IL22RA2 in published human array datasets (accession numbers: GSE5058 and GSE27597) [20-22] was performed using Array Studio software (OmicSoft Corporation, Research Triangle Park, NC, USA).

Affiliations: ${ }^{1}$ Priority Research Centres GrowUpWell and Healthy Lungs, School of Biomedical Sciences and Pharmacy, Hunter Medical Research Institute and University of Newcastle, Callaghan, Australia. ${ }^{2}$ Interdepartmental Study Center for Inflammatory and Smoke-related Airway Diseases (CEMICEF), Cardiorespiratory and Internal Medicine Section, University of Ferrara, Ferrara, Italy. ${ }^{3}$ The Airways Disease Section, National Heart and Lung Institute, Imperial College London, London, UK. ${ }^{4}$ The University of British Columbia Center for Heart Lung Innovation, St Paul's Hospital, Vancouver, BC, Canada. ${ }^{5}$ Respiratory Division, Dept of Medicine, University of British Columbia, Vancouver, BC, Canada. ${ }^{6}$ Centre for inflammation, Centenary Institute, Sydney, Australia. ${ }^{7}$ School of Life Sciences, University of Technology, Ultimo, Australia. ${ }^{8}$ Laboratory of Immunoregulation, Cancer and Inflammation Program, Center for Cancer Research, National Cancer Institute, National Institutes of Health, Frederick, MD, USA. ${ }^{9}$ UOC di Pneumologia, Dipartimento di Scienze Biomediche, Odontoiatriche e delle Immagini Morfologiche e Funzionali (BIOMORF), Università di Messina, Messina, Italy.

Correspondence: Philip M. Hansbro, Centre for Inflammation, Centenary Institute, Sydney, and School of Life Sciences, University of Technology, Ultimo, NSW, Australia. E-mail: p.hansbrođcentenary.org.au 
Mice

Animals were female, 7-8-week-old, WT C57BL/6 mice, Ill7 $a^{e G F P /+} ; I l 22^{t d-t o m a t o /+}$ reporter and $I l 22^{-/-}$ mice on a C57BL/6 background [19].

Experimental COPD

Mice were exposed to normal air or nose-only inhalation of CS for 8 weeks in a protocol representative of a pack-a-day smoker as extensively described previously $[4,12-18,23,24]$.

\section{Quantitative PCR}

Total RNA was extracted from whole lung tissue and blunt-dissected airways and parenchyma and reverse transcribed [13]. mRNA transcripts were determined using real-time quantitative (q) PCR (ABIPrism7000, Applied Biosystems, Scoresby, Victoria, Australia) using custom-designed primers (Integrated DNA Technologies, Baulkham Hills, New South Wales, Australia) (supplementary table S1).

Flow cytometry

IL-17A ${ }^{+}$and IL-22 ${ }^{+}$CD4 ${ }^{+}$T-cells, $\gamma \delta$ T-cells, NKT-cells and ILC3s in lung homogenates were determined based on surface marker expression (supplementary table S2) [25-27] using a BD FACSAriaIII. Flow cytometry antibodies were from Biolegend (Karrinyup, Australia) or BD Biosciences (North Ryde, Australia) (supplementary table S3, supplementary figure S1).

\section{Pulmonary inflammation}

Airway inflammation was assessed by differential enumeration of inflammatory cells in bronchoalveolar lavage fluid (BALF) $[12,14,28,29]$. BALF supernatants were stored at $-20^{\circ} \mathrm{C}$ for assessment of IL-22 protein levels. Tissue inflammation was assessed by enumeration of inflammatory cells [12-14, 29] and histopathological scoring based on established criteria [30].

ELISA

IL-17A, IL-22, myeloperoxidase (MPO) and neutrophil elastase protein levels were quantified using commercially available ELISA kits (R\&D Systems or Biolegend) [19].

Immunohistochemistry

Lungs were perfused, inflated, formalin fixed, paraffin embedded and sectioned $(4 \mu \mathrm{m})[13,14]$. Longitudinal sections of the left lung were deparaffinised and stained with antibodies against IL-22RA1 or IL-22RA2. Immunohistochemistry (IHC) in human samples is described in supplementary tables S4-S6 [31].

Airway remodelling

Airway epithelial $\left(\mu \mathrm{m}^{2}\right)$ and collagen deposition area $\left(\mu \mathrm{m}^{2}\right)$ were assessed in a minimum of four small airways (basement membrane perimeter $<1000 \mu \mathrm{m}$ ) per section $[12-14,17,18]$. Data were quantified using Image software (version 1.50; National Institutes of Health, Bethesda, MD, USA) and normalised to basement membrane perimeter $(\mu \mathrm{m})$.

\section{Alveolar enlargement}

Alveolar diameter was assessed using the mean linear intercept technique $[12-14,17,18,32]$.

\section{Lung function}

Mice were anaesthetised using ketamine $\left(100 \mathrm{mg} \cdot \mathrm{kg}^{-1}\right)$ and xylazine $\left(10 \mathrm{mg} \cdot \mathrm{kg}^{-1}\right)$, tracheas cannulated and attached to Buxco ${ }^{\circ}$ Forced Maneuvres apparatus (DSI, St. Paul, MN, USA) to assess total lung capacity (TLC) $[12,13]$. FlexiVent apparatus (FX1 System; SCIREQ, Montreal, Canada) was used to assess lung volume, airway resistance, inspiratory capacity, forced vital capacity (FVC), compliance and elastance (tidal volume $8 \mathrm{~mL} \cdot \mathrm{kg}^{-1}$, respiratory frequency 450 breaths $\left.\cdot \mathrm{min}^{-1}\right)[12,33,34]$.

\section{Results}

IL-22 and IL-22R mRNA expression and protein levels are increased in human COPD

First, we determined whether the mRNA expression of IL-22 and its receptors IL-22RA1 and IL-10RB and antagonist IL-22RA2 were altered in humans with mild-to-moderate COPD (Global Initiative for Chronic Obstructive Lung Disease (GOLD) stage I or II accession GSE5058 [20, 21, 35]). Pre-existing microarray data from airway epithelial brushings of healthy nonsmokers, healthy smokers and COPD patients were interrogated [20]. IL-22, IL-22RA1, IL-10RB and IL-22RA2 mRNA expression were not significantly altered in airway epithelial brushings from healthy smokers compared to nonsmokers (figure 1a-d). However, importantly, IL-22 (2.01-fold), IL-22RA1 (2.48-fold), IL-10RB (3.26-fold) and IL-22RA2 
(1.78-fold) mRNA expression was increased in airway epithelial brushings from patients with mild-to-moderate COPD compared to nonsmokers. Similar results were observed when mild-to-moderate COPD was compared to healthy smokers.
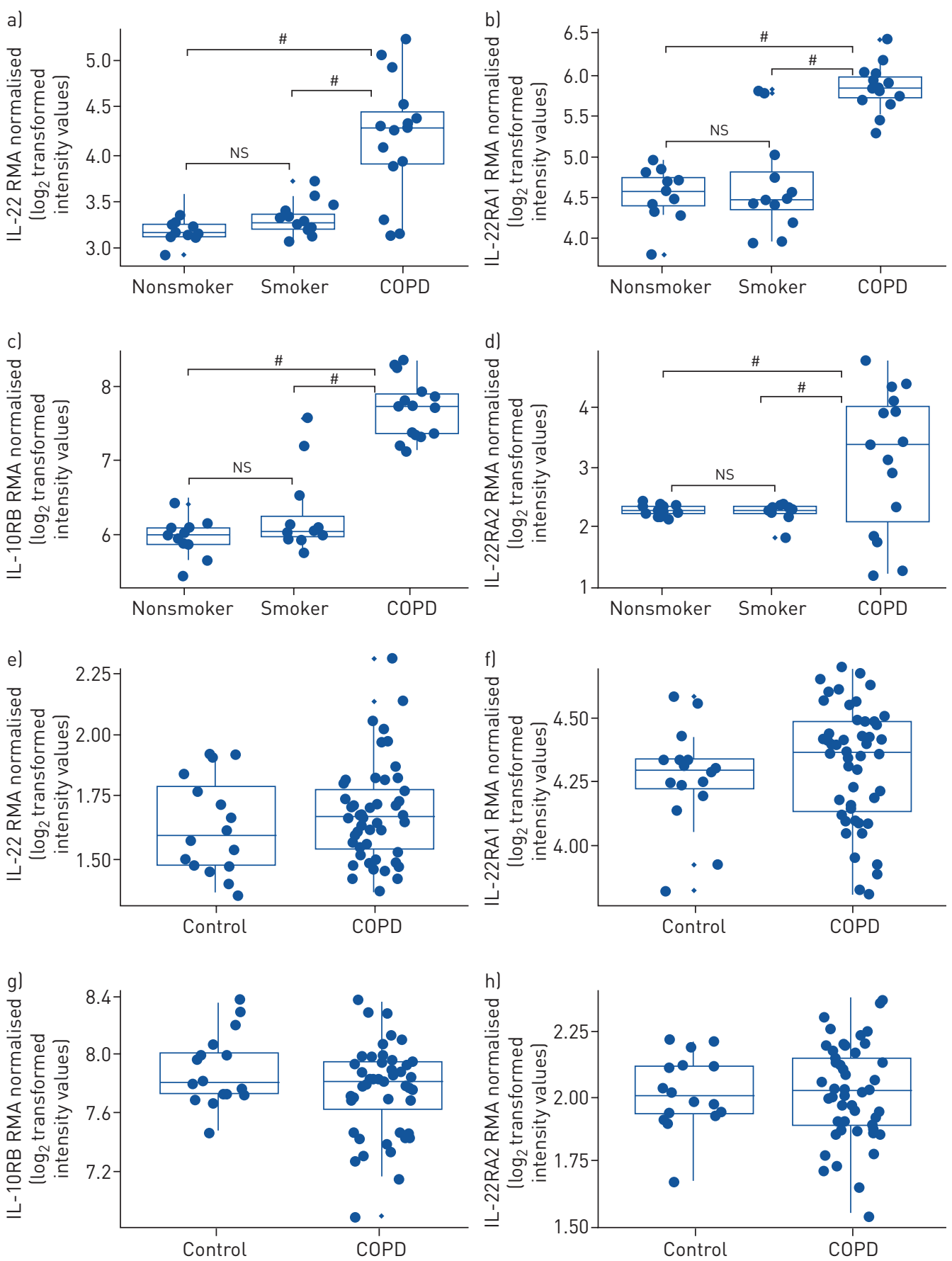

FIGURE 1 Interleukin (IL)-22 and IL-22R mRNA expression are increased in airway epithelial brushings from human mild-to-moderate chronic obstructive pulmonary disease (COPD) patients compared to healthy smokers and nonsmokers. Microarray data from airway epithelial cells from healthy human nonsmokers, healthy smokers without COPD and COPD patients with Global Initiative for Chronic Obstructive Lung Disease (GOLD) stage I (mild) or II (moderate) disease laccession: GSE5058 [20]) were interrogated: a) IL-22, b) IL-22RA1, c) IL-10RB, d) IL-22RA2 mRNA expression. Microarray data from lung parenchymal cores from human healthy nonsmokers and COPD patients with GOLD stage IV (severe) disease (accession: GSE27597 [22]) were interrogated: e) IL-22, f) IL-22RA1, g) IL-10RB, h) IL-22RA2 mRNA expression. Data are expressed as $\log _{2}$ intensity robust multi-array average signals. The Benjamini-Hochberg method for adjusted $\mathrm{p}$-value/ false discovery rate was used to analyse differences between nonsmokers, smokers and COPD patients. RMA: robust multichip averaging; NS: nonsignificant. ${ }^{\#}: p<0.005$ compared to COPD. 

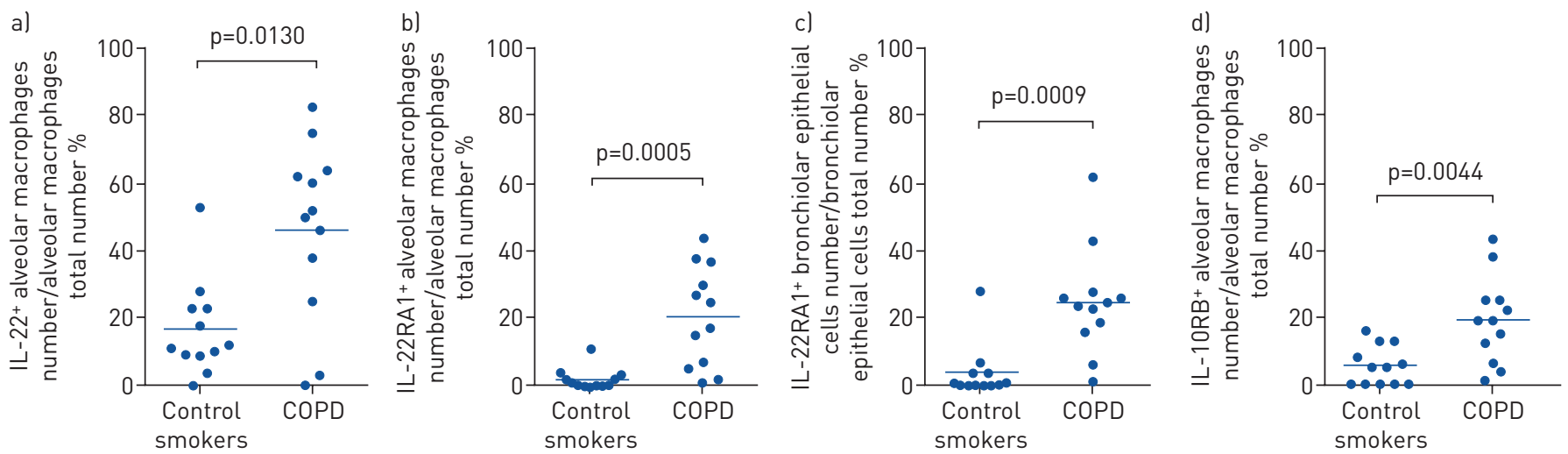

FIGURE 2 Interleukin (IL)-22, IL-22RA1 and IL-10RB, but not IL-22RA2 protein are increased in human chronic obstructive pulmonary disease (COPD). IHC for IL-22 and its receptors in peripheral lung from smokers with mild-to-moderate stable COPD and compared to age- and smoke history-matched smokers with normal lung function. a) IL-22 ${ }^{+}$alveolar macrophages; b) IL-22 receptor (IL-22R)A $1^{+}$alveolar macrophages; c) IL-22RA $1^{+}$airway epithelial cells; d) IL-10RB ${ }^{+}$alveolar macrophages. Data are presented as mean \pm SEM, $\mathrm{n}=12$ per group.

We then assessed the mRNA expression of IL-22 and its receptors in pre-existing microarray data from lung parenchyma cores from severe COPD patients (GOLD stage IV [35] accession: GSE27597 [22]). There was no change in IL-22, IL-22RA1, IL-10RB or IL-22RA2 expression in cores from COPD patients compared to nonsmokers without COPD (figure 1e-h). IL-22, IL-22RA1, IL-22RA2 and IL-10RB were unchanged in peripheral lung tissue from patients with mild emphysema (supplementary figure S2 from GSE8581). There was no significant correlation between pack-years and IL-22, IL-22RA1 and IL-22RA2 gene expression in lung tissue (supplementary figure S3 from GSE17770). Using lung cancer as a disease
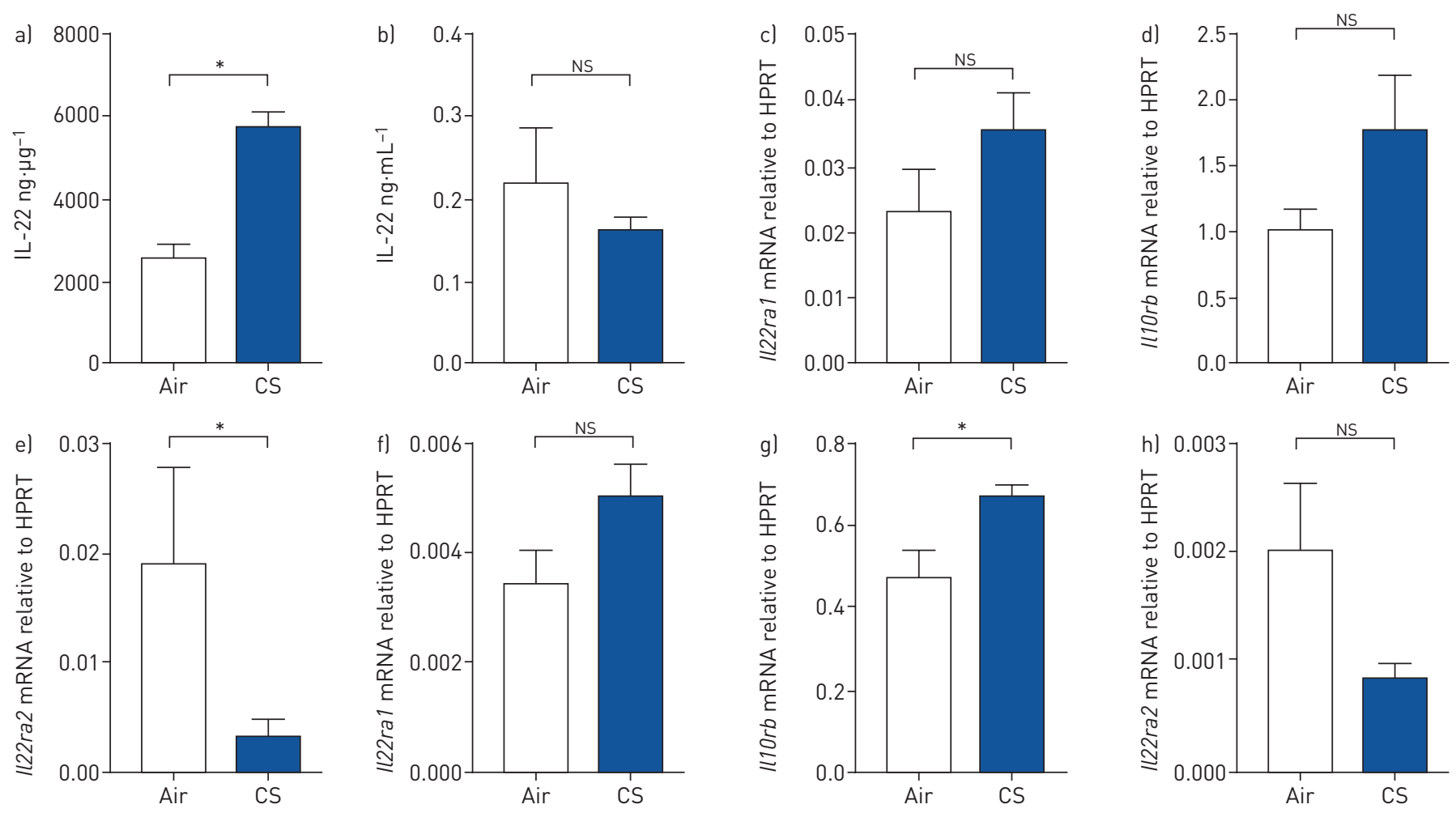

FIGURE 3 Interleukin (IL)-22 protein levels are increased in the lungs of cigarette smoke (CS)-exposed mice with experimental chronic obstructive pulmonary disease. Wild-type (WT) C57BL/6 mice were exposed to normal air or CS for 8 weeks. IL-22 protein levels in a) lung homogenates and b) bronchoalveolar lavage fluid supernatants were assessed using ELISA. In separate experiments, airways and parenchyma were blunt-dissected and IL-22 receptor mRNA expression assessed. Airway c) I(22ra1, d) /(10rb and e) /(22ra2 and parenchymal f) /(22ra1, g) /(10rb and h) /(22ra2 mRNA expression. Data are presented as mean $\pm \mathrm{SEM}, \mathrm{n}=6$, with another independent experiment showing similar results. Two-tailed Mann-Whitney t-test was used to analyse differences between two groups. *: $p<0.05$ compared to normal air-exposed WT controls; Ns: nonsignificant. 

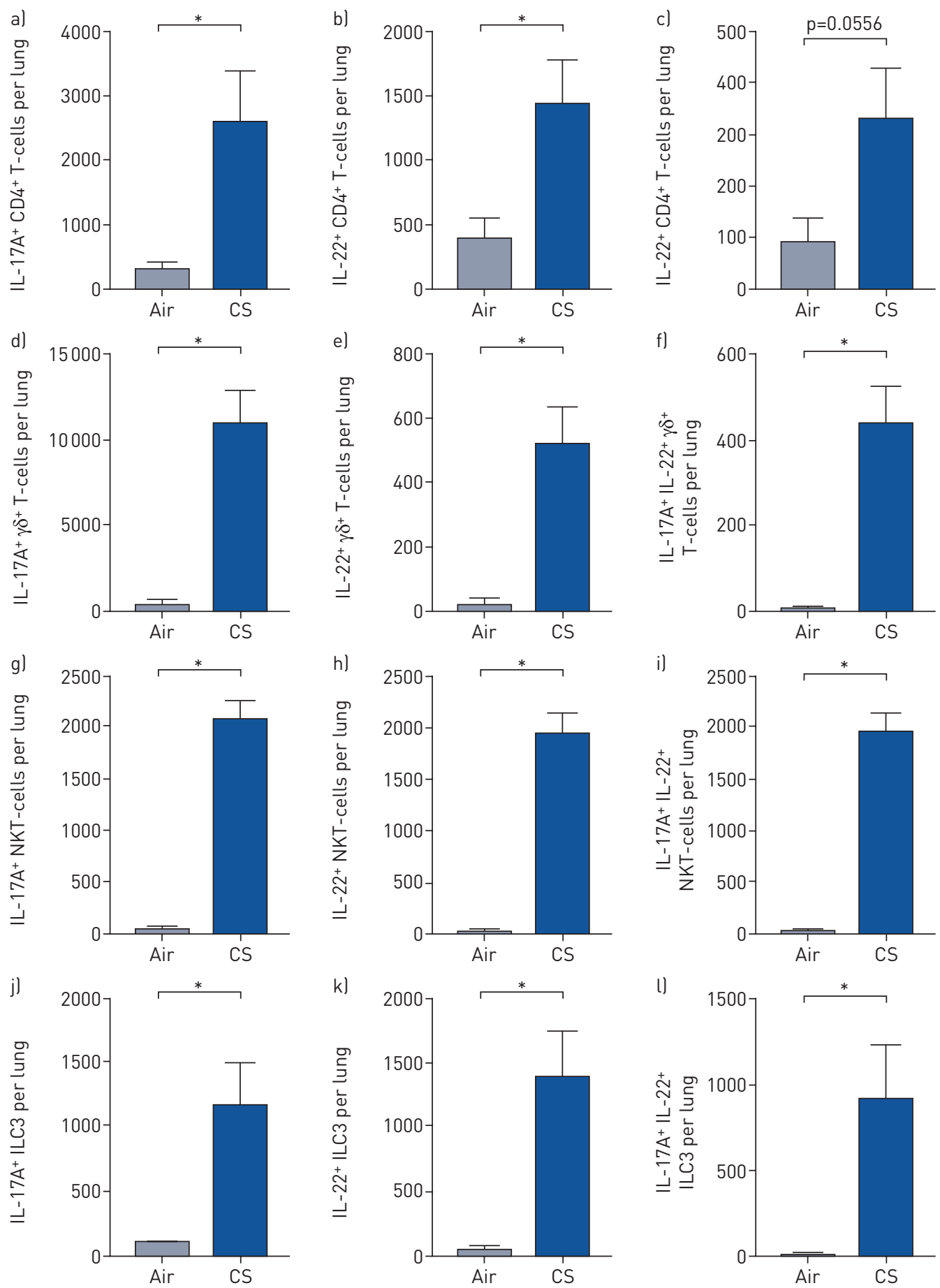

m]

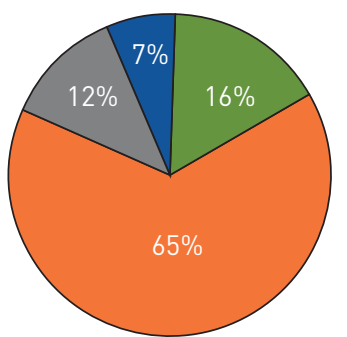

CD4+ $\mathrm{T}$-cells

n)

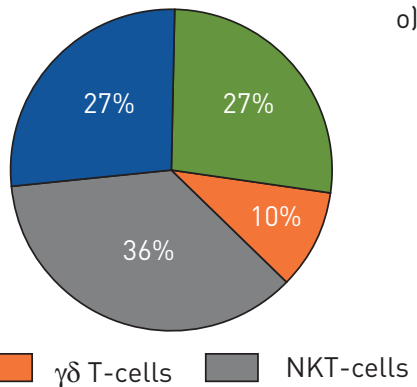

o)

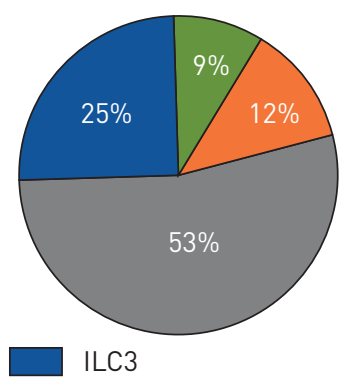


FIGURE 4 (previous page) Interleukin (IL)-22 $2^{+} \mathrm{CD}^{+} \mathrm{T}$-cells, $\gamma \delta \mathrm{T}$-cells, natural killer (NK)T-cells and group 3 innate lymphoid cells (ILC3s) are increased in the lungs of cigarette smoke (CS)-exposed mice with experimental chronic obstructive pulmonary disease. $1117 a^{e G F P /+} ; / 122^{t d-t o m a t o /+}$ reporter mice were exposed to normal air or CS for 8 weeks and the cellular source of IL-17A and IL-22 in the lung was assessed using flow

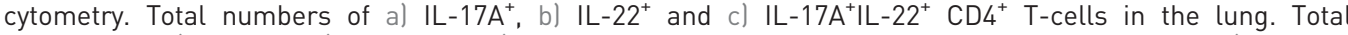

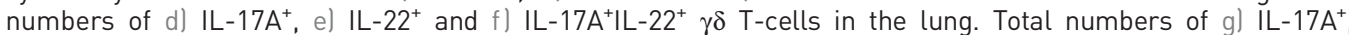

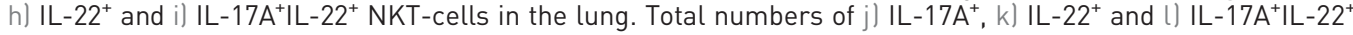
ILC3 cells in the lung. Relative proportions of CD4 ${ }^{+}$T-cells, $\gamma \delta$ T-cells, NKT-cells and ILC3s expressing m) IL-17A, n) IL-22 and o) IL-17 and IL-22. Data are presented as mean \pm SEM, $n=6$, with another independent experiment showing similar results. Two-tailed Mann-Whitney t-test was used to analyse differences between two groups. *: $\mathrm{p}<0.05$ compared to normal air-exposed controls. Representative fluorescence-activated cell

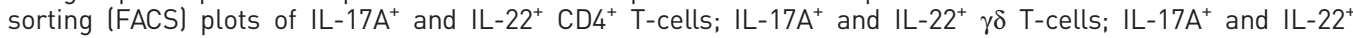
NKT-cells; and IL-17A ${ }^{+}$and IL-22+ ILC3s are presented in supplementary figure S10.

control, no differential expression of IL-22, IL-22RA1, IL-22RA2 or IL-10RB in either bronchial brushings (GSE4115) or lung tissue (GSE1650) between healthy smokers and subjects with lung cancer were observed (supplementary figures S4 and S5).

Finally, we assessed IL-22 and receptor protein levels in human COPD using IHC. The percentage of $\mathrm{IL}_{-} 22^{+}$alveolar macrophages and IL-22RA1 ${ }^{+}$and $\mathrm{IL}-10 \mathrm{RB}^{+}$airway epithelial cells were increased in COPD compared to age- and smoking history-matched smokers with normal lung function (figure 2, supplementary figure S6 and supplementary tables S6-S9). No change in IL-22RA2 was detected (supplementary table S8).

In a separate cohort of COPD patients, IL-22RA1 was increased in airway epithelial cells of current smokers with COPD compared to nonsmokers (supplementary figure S7 and supplementary table S10). When combined with ex-smokers with COPD, this IL-22RA1 signal in the airway epithelium is lost (supplementary figure S7).

\section{IL-22 and receptor protein levels are increased in the lungs in experimental COPD}

Next, we investigated the expression of IL-22 and its receptors in CS-induced experimental COPD, which models mild-to-moderate COPD. We first confirmed that IL-22 was increased in experimental COPD. Il22 mRNA was difficult to detect in mouse lungs; therefore, we assessed protein levels using ELISA in both whole-lung homogenates (include both airways and parenchyma) and BALF supernatants. CS-exposure of WT mice resulted in increased IL-22 protein levels in lung homogenates, but not BALF supernatants compared to normal air-exposed controls (figure 3a and b). IL-22 protein levels were unaltered following 1 week of CS exposure (supplementary figure S8). Collectively, these data show that IL-22 is increased in both human and experimental COPD and are consistent with previous reports [8].

Next, we assessed IL-22 receptor expression in blunt-dissected airways versus parenchymal tissue [13]. CS-exposure had no statistically significant effect on Il22ral or Il10rb mRNA expression, but did reduce Il22ra2 expression in the airways compared to normal air-exposed controls (figure $3 \mathrm{c}-\mathrm{e}$ ). CS exposure also did not affect Il22ra1 or Il22ra2 mRNA expression, but did increase Il10rb expression in the parenchyma compared to normal air-exposed controls (figure $3 \mathrm{f}-\mathrm{h}$ ). While no statistically significant differences in Il22ral mRNA expression were observed in this model, it is notable that Il22ral mRNA expression was $\sim 10$-fold higher in the airways than the parenchyma.

Finally, we assessed IL-22 receptor protein expression in mouse lung tissue sections. CS-exposure resulted in notable increases in both IL-22RA1 and IL-22RA2 protein levels, particularly in airway epithelial cells, but also in alveolar macrophages (supplementary figure S9).

\section{IL-22+ $\mathrm{CD4}^{+} T$-cells, $\gamma \delta$ T-cells, NKT-cells and ILC3s are increased in the lungs in experimental COPD}

Given that IL-22 is increased in both human and experimental COPD, we defined the cellular source of increased pulmonary IL-22 using $I l 17 a^{e G F P /+}$; Il22 $2^{\text {td-tomato/+ }}$ reporter mice which enable the detection of IL-17A ${ }^{+}$and IL- $22^{+}$cells without ex vivo stimulation. CS-exposure of reporter mice resulted in increased numbers of IL-17A ${ }^{+}$, IL- $22^{+}$and IL-17A ${ }^{+} \mathrm{IL}-22^{+} \mathrm{CD} 4^{+} \mathrm{T}$-cells, $\gamma \delta$ T-cells, NKT-cells and ILC3s compared to normal air-exposed controls (figure $4 \mathrm{a}-\mathrm{p}$ ). We then assessed the relative proportions of these cells following CS-exposure (figure 4q-s). As shown previously [36], $\gamma \delta$ T-cells were the dominant source of IL-17A following CS exposure (figure 4q). CD4 ${ }^{+}$T-cells, NKT-cells and ILC3s were the major IL-22-producing cells (figure 4r), while NKT-cells were the dominant source of dual IL-17A ${ }^{+} \mathrm{IL}_{-} 22^{+}$cells (figure $4 \mathrm{~s}$ ). 
CS-induced pulmonary neutrophils were reduced in II22-/- mice

We next investigated whether IL-22 plays a role in the pathogenesis of experimental COPD. WT and $\mathrm{Il}_{22} 2^{-/-}$mice were exposed to normal air or CS for 8 weeks [12-18]. Pulmonary inflammation in BALF was assessed by staining and differential enumeration of inflammatory cells. CS exposure of WT mice resulted in significantly increased total leukocytes, macrophages, neutrophils and lymphocytes compared to normal air-exposed WT controls (figure $5 \mathrm{a}-\mathrm{d}$ ). CS-exposed $I l 22^{-/-}$mice also had increased numbers of these cells compared to normal air-exposed $I l 22^{-/-}$controls. Neutrophils were significantly reduced, but total leukocytes, macrophages and lymphocytes were unaltered in CS-exposed $I l 22^{-1-}$ mice compared to CS-exposed WT controls.

We then assessed inflammatory cell numbers in lung tissue sections [12-14, 29]. CS exposure of WT mice significantly increased inflammatory cell numbers in the parenchyma compared to normal air-exposed WT controls (figure 5e-f). CS-exposed $1722^{-/-}$mice had increased parenchymal inflammatory cells compared to their normal air-exposed controls. Numbers of parenchymal inflammatory cells were not different between CS-exposed $I l 22^{-/-}$and WT mice.

Next, histopathology was scored according to a set of custom-designed criteria as described previously [30]. CS exposure of WT mice increased histopathology score, which was characterised by increased airway, vascular and parenchymal inflammation (figure $5 \mathrm{~g}-\mathrm{k}$ ). CS-exposed $I l 22^{-1-}$ mice had increased histopathology, airway, vascular and parenchymal inflammation scores compared to their normal air-exposed controls. Il $22^{-1-}$ mice had a small but significant reduction in total histopathology score, compared to CS-exposed WT controls.

We then profiled the mRNA expression of chemokines and cytokines, other than IL-22, that are involved in neutrophil influx into the lung including chemokine (C-X-C motif) ligand (CXCL)1, CXCL2 and IL-17A [37]. CS-exposure of WT mice resulted in significantly increased Cxcl1, Cxcl2 and Il17a mRNA expression compared to normal air-exposed WT controls with Cxcl1 and Cxcl2 having 200-fold greater expression than $\mathrm{Il17a}$ (figure 5l-n). CS-exposed $\mathrm{I} l 22^{-/-}$mice also had increased expression of Cxcl1 and $I l 17 a$, but not $\mathrm{Cxcl}$, compared to normal air-exposed $\mathrm{Il} 22^{-1-}$ controls. There was a significant reduction in Cxcl2, but not Cxcl1 or Il17a mRNA expression in CS-exposed $I l 22^{-1-}$ mice compared to CS-exposed WT controls. Protein levels of IL-17A, MPO and neutrophil elastase were increased in CS-exposed WT mice, but were unaltered in $I l 22^{-1-}$ mice (supplementary figure S11).

CS-induced increases in airway epithelial area, collagen deposition and emphysema-like alveolar enlargement do not occur in II22-/- mice

We have previously shown that CS-exposed WT mice develop small airway remodelling (increased epithelial area), fibrosis (collagen deposition) and emphysema-like alveolar enlargement after 8 weeks of CS exposure [12-14, 17, 18, 32]. Thus, we determined whether IL-22 contributes to these disease features. In agreement with our previous studies, CS exposure of WT mice increased small airway epithelial cell area compared to normal air-exposed WT controls (figure $6 \mathrm{a}$ and b). In contrast, CS-exposed Il22 ${ }^{-1-}$ mice had no change in airway epithelial cell area compared to normal air-exposed $I l 22^{-/-}$controls.

CS-exposed WT mice had increased collagen deposition compared to normal air-exposed WT controls (figure $6 \mathrm{c}$ and d). However, CS-exposed $I l 22^{-/-}$mice did not have increased collagen deposition compared to $I l 22^{-/-}$normal air-exposed controls.

CS-exposed WT mice had significantly increased alveolar diameter compared to normal air-exposed WT controls (figure $6 \mathrm{e}$ and f). CS-exposed $1722^{-/-}$mice did not have increased alveolar diameter compared normal air-exposed $I l 22^{-1-}$ controls.

As a result of the relatively small differences in airway epithelial area, collagen deposition and alveolar diameter the differences were not statistically different between CS-exposed $I l 22^{-1-}$ mice and CS-exposed WT controls.

\section{CS-induced lung function impairment is improved in II22-/- mice}

We assessed the role of IL-22 in CS-induced impairment of lung function, measured in terms of lung volume, airway resistance, TLC, inspiratory capacity, FVC and compliance. CS-exposed WT mice had increases in all of these parameters compared to normal air-exposed WT controls (figure $7 \mathrm{a}-\mathrm{f}$ ). In CS-exposed Il22 ${ }^{-1-}$ mice, none of these lung function parameters were significantly different compared to normal air-exposed $I l 22^{-/-}$ controls. Again, likely due to small changes in mild-to-moderate experimental COPD, these lung function parameters were not significantly altered in CS-exposed $I l 22^{-/-}$mice compared to CS-exposed WT controls. However, CS-exposed Il22 $2^{-1-}$ mice had similar lung function to air-exposed WT controls.

We assessed tissue elastance and found a nonsignificant reduction in CS-exposed WT mice that was not different in $I l 22^{-/-}$mice (supplementary figure S12). 

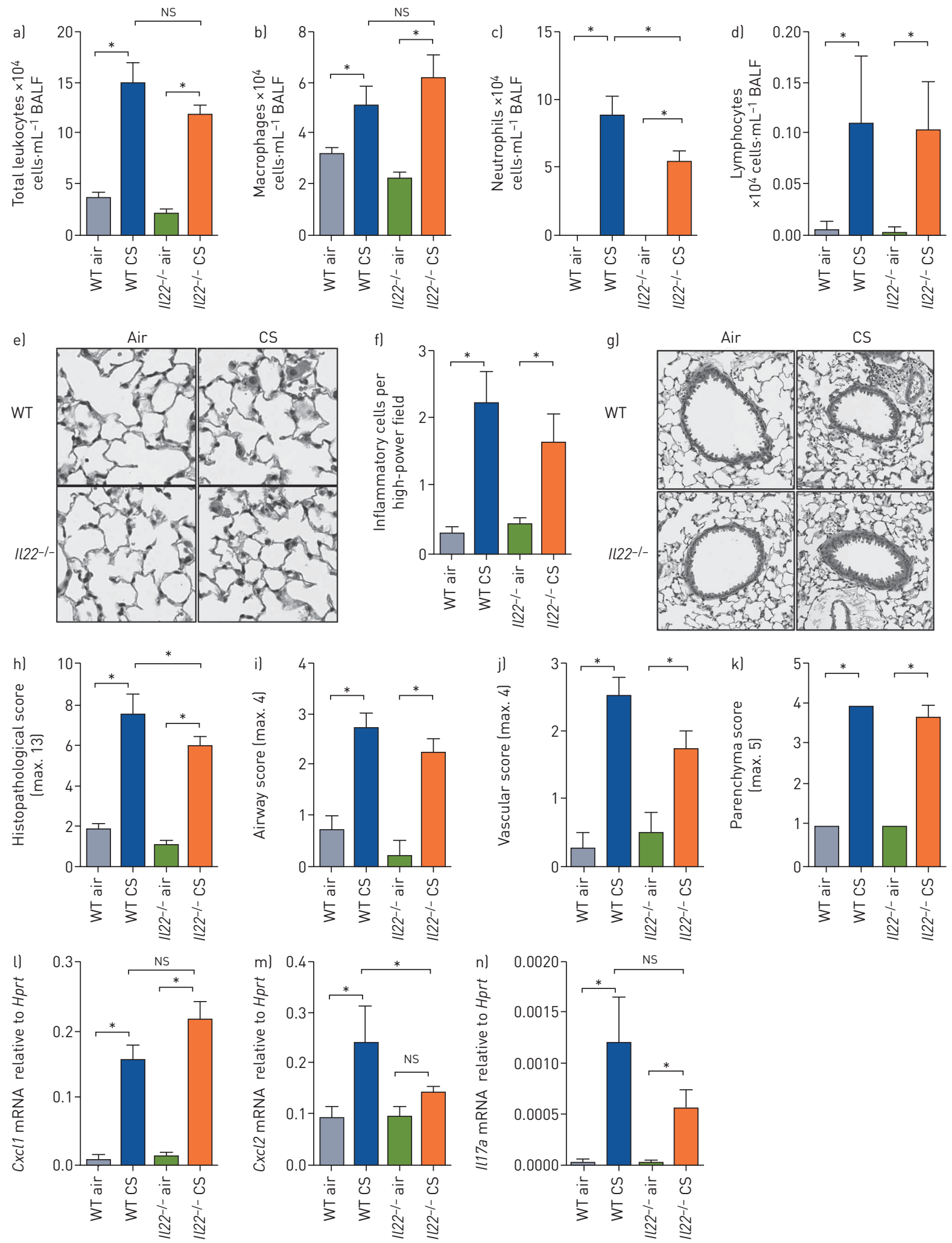

FIGURE 5 Cigarette smoke (CS)-induced pulmonary inflammation is reduced in $/ 122^{-/-}$mice. Wild-type (WT) and interleukin (IL)-22-deficient $\left(/ 122^{-/-}\right)$C57BL/6 mice were exposed to normal air or CS for 8 weeks to induce experimental chronic obstructive pulmonary disease. a) Total leukocytes, b) macrophages, c) neutrophils and d) lymphocytes in bronchoalveolar lavage fluid (BALF); e) representative images of parenchymal inflammatory cells; f) numbers of parenchymal inflammatory cells per high powered field; g) representative images of lung histopathology scoring; h) total histopathology score in lung sections and scores specifically in the i) airway, j) vascular and kJ parenchymal regions; (I) Cxcll, m) Cxcl2 and n) II17a mRNA expression in lung homogenates. Data are presented as mean \pm SEM, $n=6$, with another independent experiment showing similar results. The one-way ANOVA with Bonferroni post-test analysed differences between three or more groups. *: $p<0.05$ compared to normal air-exposed controls; NS: nonsignificant. 
a)

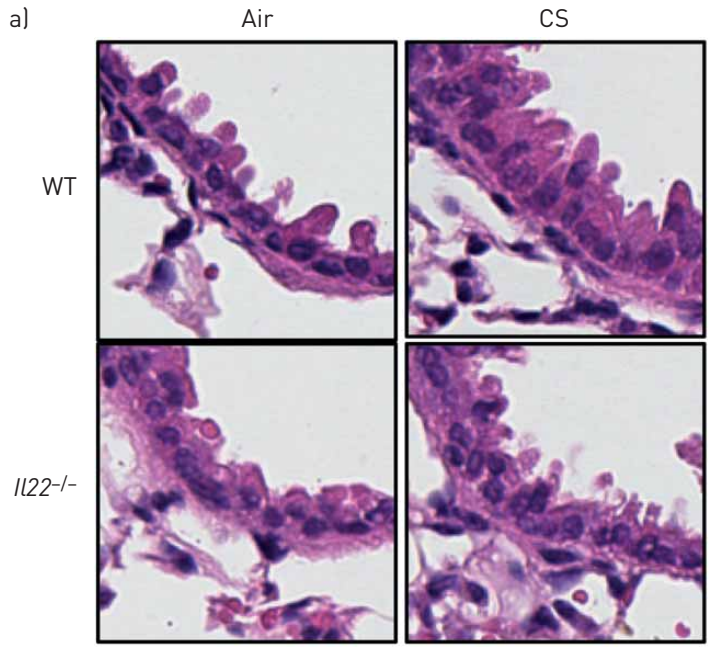

c)

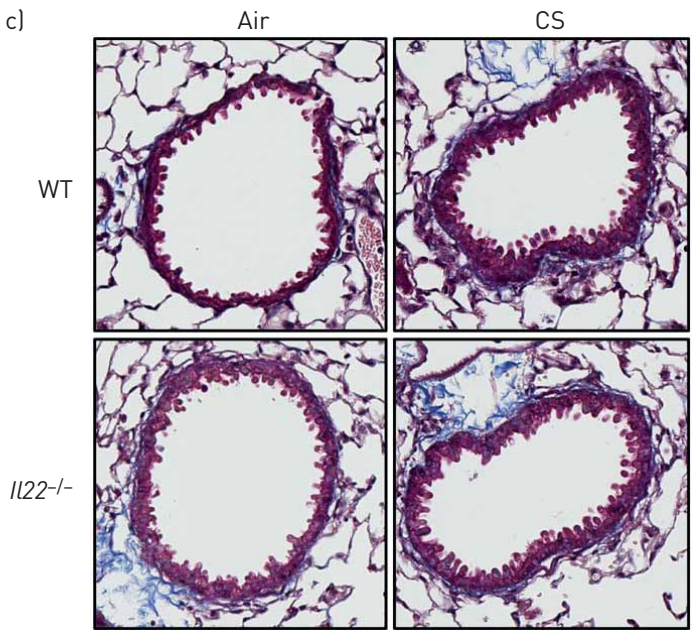

e)

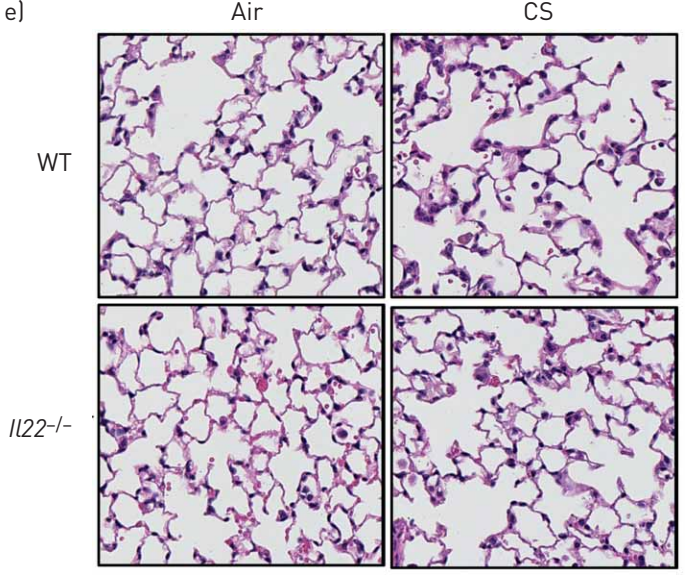

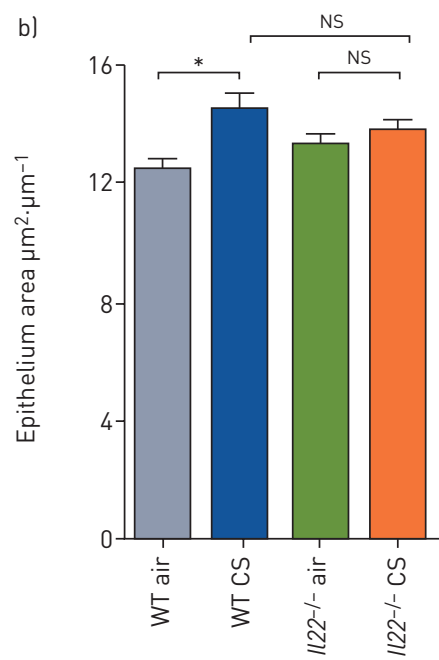

d)

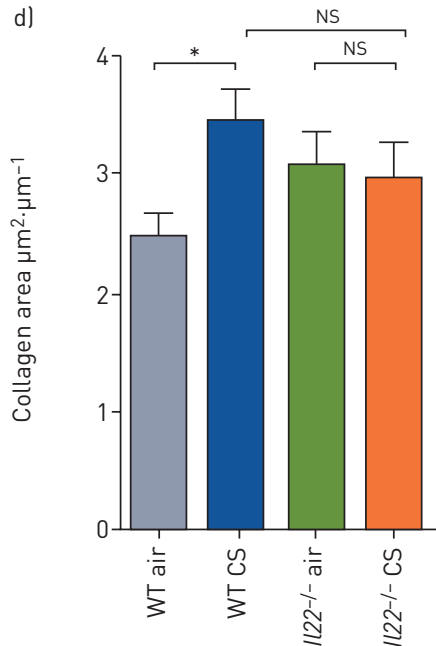

f)

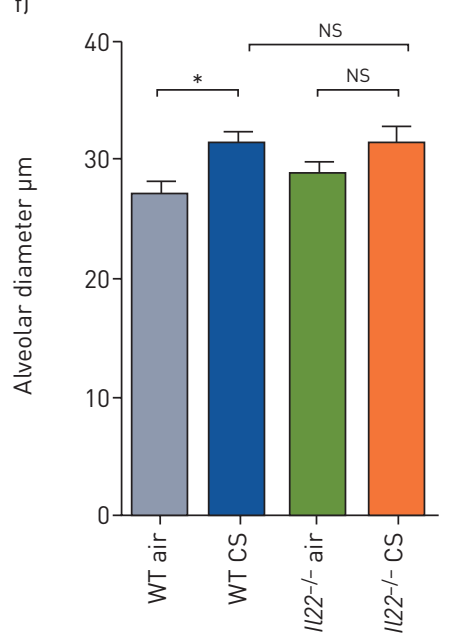

FIGURE 6 Cigarette smoke (CS)-induced increases in airway epithelial area, collagen deposition and emphysema-like alveolar enlargement do not occur in $1122^{-1-}$ mice. Wild-type (WT) and interleukin (IL)-22-deficient (//22 $\left.{ }^{-/}\right)$C57BL/6 mice were exposed to normal air or CS for 8 weeks to induce experimental chronic obstructive pulmonary disease. al Representative images of small airway epithelium; b) small airway epithelial thickness in terms of epithelial cell area $\left(\mu \mathrm{m}^{2}\right)$ per basement membrane perimeter $(\mu \mathrm{m})$; c) representative images of collagen deposition around small airways; d) area of collagen deposition $\left(\mu m^{2}\right)$ per basement membrane perimeter $(\mu \mathrm{m})$; e) representative images of alveolar structure; f) alveolar diameter $(\mu \mathrm{m})$. Data are presented as mean \pm SEM, $n=6$, with another independent experiment showing similar results. The one-way ANOVA with Bonferroni post-test analysed differences between three or more groups. ${ }^{*}$ : $p<0.05$ compared to normal air-exposed controls; Ns: nonsignificant. 

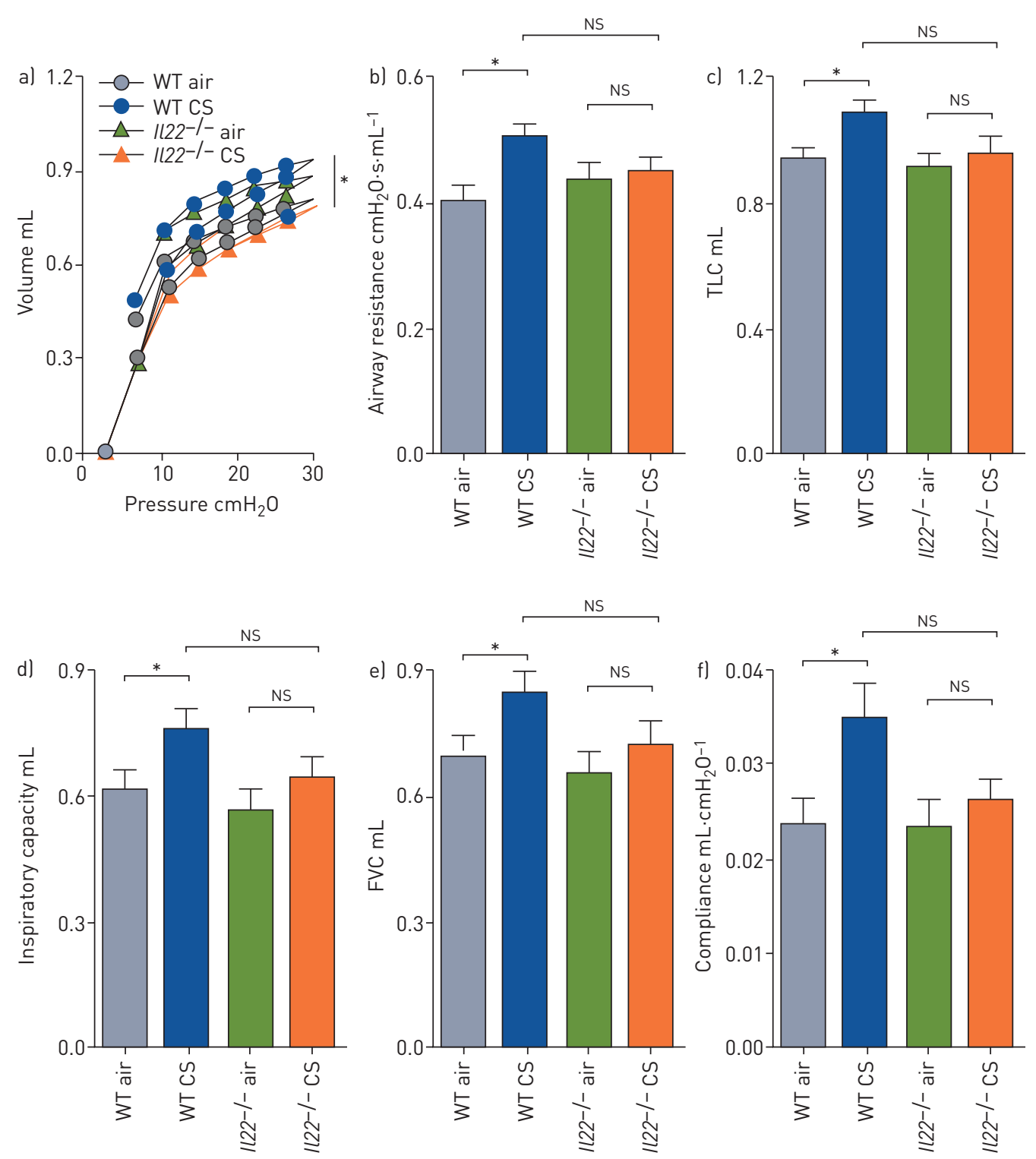

FIGURE 7 Cigarette smoke (CS)-induced lung function impairment is improved in $1122^{-/-}$mice. Wild-type (WT) and interleukin (IL)-22-deficient $\left(/ / 22^{-1-}\right)$ C57BL/6 mice were exposed to normal air or CS for 8 weeks to induce experimental chronic obstructive pulmonary disease. Lung function was assessed in terms of a) lung volume from pressure-volume loops, b) airway resistance, c) total lung capacity (TLC), d) inspiratory capacity, e) forced vital capacity (FVC) and f) compliance. Data are presented as mean \pm SEM, $n=6$, with another independent experiment showing similar results. The one-way ANOVA with Bonferroni post-test analysed differences between three or more groups. *: $p<0.05$ compared to normal air-exposed controls; NS: nonsignificant.

\section{Discussion}

Here, we demonstrate that IL-22 plays a previously undefined role in the pathogenesis of CS-induced experimental COPD. IL-22 and its receptors were increased in both human and experimental COPD. We show for the first time, using IL-22 reporter mice, that elevated lung IL-22 levels in experimental COPD result from increased IL-22 ${ }^{+} \mathrm{CD} 4^{+}$T-cells, $\gamma \delta$ T-cells, NKT-cells and ILC3s. In addition, we have demonstrated that CS-induced neutrophilic airway inflammation was reduced in $I l 22^{-/-}$mice compared to WT controls. Furthermore, Il22 ${ }^{-/-}$mice did not develop CS-induced airway remodelling and emphysema and had improved lung function that was comparable to normal air-exposed controls. Hence, this study provides new insights into the roles of IL-22 in the pathogenesis of COPD.

The presence or absence of IL-22 may affect resident microbiota. Indeed, we have reviewed the pathogenic roles for gut and lung microbiota in the development of COPD $[5,38,39]$. To minimise the influence of 
altered microbiota, WT and $1722^{-/-}$mice were derived from the same breeding pairs, maintained in the same facility and used experimentally at the same time, and so they would be expected to have very similar microbiomes.

Using pre-existing microarray datasets, we show that IL-22 and IL-22R mRNA expression were increased in airway epithelial cells from patients with mild-to-moderate COPD [20]. However, IL-22 and IL-22R mRNA were unaltered in lung parenchymal cores in severe COPD [22]. Our data are supported by studies that show increased IL-22 protein levels and IL-22 ${ }^{+}$immune cells in blood, sputum and lung biopsies of COPD patients (reviewed in [8]). However, there are limited reports of IL-22 receptor expression in COPD. Neutrophil proteases have been shown to alter IL-22R-dependent antimicrobial defence in COPD, but there was no change in IL22RA1 mRNA expression in lung tissue or primary cultures of proximal airway epithelial cells from COPD patients compared to healthy controls [9]. IL-10RB and IL-22RA2 have not been assessed in COPD. Consistent with our human data, IL-22 was increased in lung tissue homogenates in experimental COPD after 8 weeks, but not before the development of disease upon 1 week of CS exposure. IL-22 receptor mRNA expression was different between human and mouse. However, at the protein level, IL-22RA1 and RA2 were visually increased in the airway epithelium of CS-exposed mice, which was consistent with changes at the mRNA level in humans. IL-22 receptors were also increased at protein level in human COPD. Collectively, our data show that IL-22 and its receptors are increased in both human and experimental COPD. However, the expression of IL-22 and its receptors is heterogenous and is influenced by tissue location and disease severity.

Given that IL-22 was increased in the lungs in experimental COPD, we used IL-17A and IL-22 dual reporter mice that facilitate the identification of IL-17A- and IL-22-expressing immune cells without ex vivo stimulation or cell fixation. This enables a more accurate determination of the in vivo lung environment. We show for the first time that CS exposure induced IL-22 production from CD $4^{+} \mathrm{T}$-cells, $\gamma \delta$ T-cells, NKT-cells and ILC3s, which are the major cellular sources of IL-22, and all these cell subsets have known roles in COPD pathogenesis [36, 40,41]. However, the individual contribution of each of these cells to IL-22 production and COPD pathogenesis remains to be fully elucidated, especially in humans.

Previously, the role of IL-22 in the pathogenesis of COPD was largely unknown. We addressed this gap in knowledge using an established mouse model of tightly controlled chronic nose-only CS-induced experimental COPD [12-18]. Our models are representative of a pack-a-day smoker [24]. We have consistently shown that 8 weeks of CS exposure in our models is sufficient to induce the hallmark features of human COPD: chronic inflammation, airway remodelling, emphysema and impaired lung function [1218]. This 8 -week time point was specifically chosen to investigate the underlying pathogenic mechanism(s) during the early stages (GOLD I/II) and identify potential therapeutic targets to halt the progression of COPD

Using this established model, we show for the first time that IL-22 contributes to COPD pathogenesis independently of infectious exacerbations. $I l 22^{-1-}$ mice had reduced airway neutrophils, which was associated with decreased Cxcl2 mRNA expression. CXCL1 and CXCL2 are the mouse orthologues/ homologues of human IL- 8 and have critical roles in neutrophil influx into the airways following CS-exposure [42]. It has been suggested that improper activation of neutrophils lies at the core of COPD pathology, and mechanisms regulating their function are potential therapeutic targets [43]. However, Il22 $2^{-1-}$ mice were protected from the increases in MPO or neutrophil elastase levels. $I l 22^{-l-}$ mice also had decreased lung tissue inflammation indicated by reduced histopathological score. This is consistent with a previous report showing that administration of recombinant (r)IL-22 into the lung increased tissue inflammation [44].

Additionally, we demonstrate, as we have shown previously, that increases in airway epithelial area, collagen deposition around small airways and emphysema-like alveolar enlargement occur following chronic CS exposure in WT mice [12-18]. Notably, these features did not develop in Il $22^{-1-}$ mice compared to normal air-exposed $I l 22^{-/-}$controls, although the changes were not significant between CS-exposed Il22-/- mice and CS-exposed WT controls. IL-22 is essential for lung epithelial cell repair following influenza virus infection and is implicated in renal fibrosis $[45,46]$. Others have shown that mice lacking IL-22 have delayed bacterial clearance and increased alveolar wall thickening and airway remodelling [10]. Administration of rIL-22 with or without acute CS-exposure induced airway epithelial thickening and collagen deposition, although this was not quantified [44].

Our study is the first report on the role of IL-22 in regulating multiple lung function parameters, particularly in models of COPD. We show that $I l 22^{-1-}$ mice have improved lung function in terms of lung volumes, airway resistance, TLC, inspiratory capacity, FVC and compliance, comparable to normal air-exposed WT mice. One previous report in an acute CS-exposure model showed increased airway resistance following administration of rIL-22 [44]; however, ours is the first study to assess lung function in $\mathrm{Il} 22^{-1-}$ mice. 
The absence of IL-22 in CS-exposed $I l 22^{-/-}$mice suppressed both airway remodelling and concomitantly the impairment of lung function in experimental COPD. Indeed, CS-exposed $I l 22^{-/-}$mice were protected against increases in epithelial area, collagen deposition and emphysema compared to normal air-exposed controls. Airway remodelling involving epithelial hyperplasia and fibrosis are important in driving resistance to airflow $[17,18]$. Emphysema leads to apparent increases in total lung and inspiratory capacity and tissue compliance, which results from the loss of alveolar and parenchymal tissue. In line with the protection against airway remodelling and emphysema-like alveolar enlargement, CS-exposed $I l 22^{-/-}$mice were protected from impaired lung function and changes in airway resistance, total lung and inspiratory capacities and tissue compliance.

In summary, our study demonstrates previously unrecognised roles for IL-22 in COPD pathogenesis. It highlights the potential role of IL-22 in chronic lung diseases, which may be a useful biomarker in the diagnosis and/or prognosis of COPD patients. Furthermore, using a clinically relevant and established model of experimental COPD, our study demonstrates that IL-22 promotes CS-induced pulmonary neutrophilic inflammation, airway remodelling and lung function impairment. However, inhibiting IL-22 may increase the risk of exacerbations due to its central role in pathogen clearance. Therefore, caution in therapeutic approaches targeting IL-22 signalling are required. The relationships between IL-22 and genetic factors, infections/colonisation and phenotypes in COPD remain to be defined.

Acknowledgements: We acknowledge Dale Godfrey (University of Melbourne, Melbourne, Australia) for $\alpha$ GalCer tetramers, Kristy Wheeldon and Nathalie Kiaos for CS exposure of mice, Tegan Moore for assistance with data generation and Jessica Weaver for assistance with $I l 22^{-/-}$and reporter mouse colonies (All at the University of Newcastle, Newcastle, Australia).

Support statement: This study was supported by grants from the National Health and Medical Research Council (NHMRC) of Australia and the Australian Research Council (ARC), The University of Newcastle and Hunter Medical Research Institute. M.R. Starkey was supported by an NHMRC Early Career Research Fellowship and is supported by an ARC Discovery Early Career Researcher Award (DECRA) fellowship. C. Donovan is supported by an NHMRC Early Career Research Fellowship. I.M. Adcock is supported by a Wellcome Trust grant. P.M. Hansbro is supported by an NHMRC Principal Research Fellowship (1079187) and by a Brawn Fellowship, Faculty of Health and Medicine, the University of Newcastle. Funding information for this article has been deposited with the Crossref Funder Registry.

Conflict of interest: M.R. Starkey has nothing to disclose. M.W. Plank is a full-time employee of GlaxoSmithKline. P. Casolari has nothing to disclose. A. Papi reports board membership, consultancy, payment for lectures, grants for research and travel expenses reimbursement from Chiesi, AstraZeneca, GlaxoSmithKline, Boehringer Ingelheim, Mundipharma and TEVA, payment for lectures and travel expenses reimbursement from Menarini, Novartis, Zambon and Sanofi, outside the submitted work. S. Pavlidis has nothing to disclose. Y Guo has nothing to disclose. G.J.M. Cameron has nothing to disclose. T.J. Haw has nothing to disclose. A. Tam has nothing to disclose. M. Obeidat has nothing to disclose. C. Donovan has nothing to disclose. N.G. Hansbro has nothing to disclose. D.H. Nguyen has nothing to disclose. P.M. Nair has nothing to disclose. R.Y. Kim has nothing to disclose. J.C. Horvat has nothing to disclose. G.E. Kaiko has nothing to disclose. S.K. Durum has nothing to disclose. P.A. Wark has nothing to disclose. D. D. Sin reports grants from Merck, personal fees for advisory board work from Sanofi-Aventis and Regeneron, grants and personal fees from Boehringer Ingelheim, grants and personal fees for lecturing and advisory board work from AstraZeneca, personal fees for lecturing and advisory board work from Novartis, outside the submitted work. G. Caramori has nothing to disclose. I.M. Adcock has nothing to disclose. P.S. Foster has nothing to disclose. P.M. Hansbro reports funding/consultancies from Pharmaxis, AstraZeneca, Sanofi, Pharmakea, Ausbio, and Allakos outside the submitted work.

\section{References}

1 Lozano R, Naghavi M, Foreman K, et al. Global and regional mortality from 235 causes of death for 20 age groups in 1990 and 2010: a systematic analysis for the Global Burden of Disease Study 2010. Lancet 2012; 380: 2095-2128.

2 Keely S, Talley NJ, Hansbro PM. Pulmonary-intestinal cross-talk in mucosal inflammatory disease. Mucosal Immunol 2012; 5: 7-18.

3 Barnes PJ. Corticosteroid resistance in patients with asthma and chronic obstructive pulmonary disease. J Allergy Clin Immunol 2013; 131: 636-645.

4 Fricker M GB, Mateer S, Jones B, et al. Chronic smoke exposure induces systemic hypoxia that drives intestinal dysfunction. JCI Insight 2018; 3: 94040.

5 Budden KF, Gellatly SL, Wood DL, et al. Emerging pathogenic links between microbiota and the gut-lung axis. Nat Rev Microbiol 2017; 15: 55-63.

6 Dudakov JA, Hanash AM, van den Brink MR. Interleukin-22: immunobiology and pathology. Annu Rev Immunol 2015; 33: 747-785.

$7 \mathrm{Xu} \mathrm{W}$, Presnell SR, Parrish-Novak J, et al. A soluble class II cytokine receptor, IL-22RA2, is a naturally occurring IL-22 antagonist. Proc Natl Acad Sci USA 2001; 98: 9511-9516.

8 Le Rouzic O, Pichavant M, Frealle E, et al. Th17 cytokines: novel potential therapeutic targets for COPD pathogenesis and exacerbations. Eur Respir J 2017; 50: 1602434.

9 Guillon A, Jouan Y, Brea D, et al. Neutrophil proteases alter the interleukin-22-receptor-dependent lung antimicrobial defence. Eur Respir J 2015; 46: 771-782.

10 Sharan R, Perez-Cruz M, Kervoaze G, et al. Interleukin-22 protects against non-typeable Haemophilus influenzae infection: alteration during chronic obstructive pulmonary disease. Mucosal Immunol 2017; 10: 139-149. 
11 Pichavant M, Sharan R, Le Rouzic O, et al. IL-22 defect during Streptococcus pneumoniae infection triggers exacerbation of chronic obstructive pulmonary disease. EBioMedicine 2015; 2: 1686-1696.

12 Beckett EL, Stevens RL, Jarnicki AG, et al. A new short-term mouse model of chronic obstructive pulmonary disease identifies a role for mast cell tryptase in pathogenesis. J Allergy Clin Immunol 2013; 131: 752-762.

13 Haw TJ, Starkey MR, Pavlidis S, et al. Toll-like receptor 2 and 4 have opposing roles in the pathogenesis of cigarette smoke-induced chronic obstructive pulmonary disease. Am J Physiol Lung Cell Mol Physiol 2018; 314: L298-L317.

14 Haw TJ, Starkey MR, Nair PM, et al. A pathogenic role for tumor necrosis factor-related apoptosis-inducing ligand in chronic obstructive pulmonary disease. Mucosal Immunol 2016; 9: 859-872.

15 Hsu AC, Starkey MR, Hanish I, et al. Targeting PI3K-p110 $\alpha$ suppresses influenza virus infection in chronic obstructive pulmonary disease. Am J Respir Crit Care Med 2015; 191: 1012-1023.

16 Hsu AC, Dua K, Starkey MR, et al. MicroRNA-125a and -b inhibit A20 and MAVS to promote inflammation and impair antiviral response in COPD. JCI Insight 2017; 2: e90443.

17 Liu G, Cooley MA, Jarnicki AG, et al. Fibulin-1 regulates the pathogenesis of tissue remodeling in respiratory diseases. JCI Insight 2016; 1: e86380.

18 Hansbro PM, Hamilton MJ, Fricker M, et al. Importance of mast cell Prss31/transmembrane tryptase/tryptase- $\gamma$ in lung function and experimental chronic obstructive pulmonary disease and colitis. J Biol Chem 2014; 289: 18214-18227.

19 Plank MW, Kaiko GE, Maltby S, et al. Th22 cells form a distinct Th lineage from Th17 cells in vitro with unique transcriptional properties and Tbet-dependent Th1 plasticity. J Immunol 2017; 198: 2182-2190.

20 Carolan BJ, Heguy A, Harvey BG, et al. Up-regulation of expression of the ubiquitin carboxyl-terminal hydrolase L1 gene in human airway epithelium of cigarette smokers. Cancer Res 2006; 66: 10729-10740.

21 Harvey BG, Heguy A, Leopold PL, et al. Modification of gene expression of the small airway epithelium in response to cigarette smoking. J Mol Med 2007; 85: 39-53.

22 Campbell JD, McDonough JE, Zeskind JE, et al. A gene expression signature of emphysema-related lung destruction and its reversal by the tripeptide GHK. Genome Med 2012; 4: 67.

23 Fricker M, Deane A, Hansbro PM. Animal models of chronic obstructive pulmonary disease. Expert Opin Drug Discov 2014; 9: 629-645.

24 Jones B, Donovan C, Liu G, et al. Animal models of COPD: what do they tell us? Respirology 2017; 22: 21-32.

25 Starkey MR, Essilfie AT, Horvat JC, et al. Constitutive production of IL-13 promotes early-life Chlamydia respiratory infection and allergic airway disease. Mucosal Immunol 2013; 6: 569-579.

26 Starkey MR, Nguyen DH, Essilfie AT, et al. Tumor necrosis factor-related apoptosis-inducing ligand translates neonatal respiratory infection into chronic lung disease. Mucosal Immunol 2014; 7: 478-488.

27 Kedzierski L, Tate MD, Hsu AC, et al. Suppressor of cytokine signaling (SOCS)5 ameliorates influenza infection via inhibition of EGFR signaling. eLife 2017; 6: e20444.

28 Essilfie AT, Horvat JC, Kim RY, et al. Macrolide therapy suppresses key features of experimental steroid-sensitive and steroid-insensitive asthma. Thorax 2015; 70: 458-467.

29 Nair PM, Starkey MR, Haw TJ, et al. Targeting PP2A and proteasome activity ameliorates features of allergic airway disease in mice. Allergy 2017; 72: 1891-1903.

30 Horvat JC, Beagley KW, Wade MA, et al. Neonatal chlamydial infection induces mixed T-cell responses that drive allergic airway disease. Am J Respir Crit Care Med 2007; 176: 556-564.

31 Tam A, Hughes M, McNagny KM, et al. Hedgehog signaling in the airway epithelium of patients with chronic obstructive pulmonary disease. Sci Rep 2019; 9: 3353.

32 Horvat JC, Starkey MR, Kim RY, et al. Early-life chlamydial lung infection enhances allergic airways disease through age-dependent differences in immunopathology. J Allergy Clin Immunol 2010; 125: 617-625.

33 Kim RY, Horvat JC, Pinkerton JW, et al. MicroRNA-21 drives severe, steroid-insensitive experimental asthma by amplifying phosphoinositide 3-kinase-mediated suppression of histone deacetylase 2. J Allergy Clin Immunol 2017; 139: 519-532.

34 Kim RY, Pinkerton JW, Essilfie AT, et al. Role for NLRP3 inflammasome-mediated, IL-1 $\beta$-dependent responses in severe, steroid-resistant asthma. Am J Respir Crit Care Med 2017; 196: 283-297.

35 Vogelmeier CF, Criner GJ, Martinez FJ, et al. Global Strategy for the Diagnosis, Management, and Prevention of Chronic Obstructive Lung Disease 2017 Report. GOLD Executive Summary. Am J Respir Crit Care Med 2017; 195: $557-582$.

36 Shan M, Yuan X, Song LZ, et al. Cigarette smoke induction of osteopontin (SPP1) mediates $\mathrm{T}_{\mathrm{H}} 17$ inflammation in human and experimental emphysema. Sci Transl Med 2012; 4: 117ra9.

37 Aujla SJ, Dubin PJ, Kolls JK. Interleukin-17 in pulmonary host defense. Exp Lung Res 2007; 33: 507-518.

38 Shukla SD, Budden KF, Neal R, et al. Microbiome effects on immunity, health and disease in the lung. Clin Transl Immunology 2017; 6: e133.

39 Budden KF, Shukla SD, Rehman SF, et al. Functional effects of the microbiota in chronic respiratory disease Lancet Respir Med 2019; in press [https://10.1016/S2213-2600(18)30510-1].

40 Pichavant M, Rémy G, Bekaert S, et al. Oxidative stress-mediated iNKT-cell activation is involved in COPD pathogenesis. Mucosal Immunol 2014; 7: 568-578.

41 De Grove KC, Provoost S, Verhamme FM, et al. Characterization and quantification of innate lymphoid cell subsets in human lung. PLoS One 2016; 11: e0145961.

42 Thatcher TH, McHugh NA, Egan RW, et al. Role of CXCR2 in cigarette smoke-induced lung inflammation. Am J Physiol Lung Cell Mol Physiol 2005; 289: L322-L328.

43 Meijer M, Rijkers GT, van Overveld FJ. Neutrophils and emerging targets for treatment in chronic obstructive pulmonary disease. Expert Rev Clin Immunol 2013; 9: 1055-1068.

$44 \mathrm{Li}$ JR, Zhou WX, Huang KW, et al. Interleukin-22 exacerbates airway inflammation induced by short-term exposure to cigarette smoke in mice. Acta Pharmacol Sin 2014; 35: 1393-1401.

45 Pociask DA, Scheller EV, Mandalapu S, et al. IL-22 is essential for lung epithelial repair following influenza infection. Am J Pathol 2013; 182: 1286-1296.

46 Wang S, Li Y, Fan J, et al. Interleukin-22 ameliorated renal injury and fibrosis in diabetic nephropathy through inhibition of NLRP3 inflammasome activation. Cell Death Dis 2017; 8: e2937. 